\title{
Adoption of Standard ERP Solution in Health Care Sector: Is SAP ERP All-in-One Capable to Meet Specific Requirements?
}

\author{
Adnan Kraljić, Tarik Kraljić, and Denis Delismajlović \\ \{akraljic,tkraljic\}@ibu.edu.ba, denisdelismajlovic@gmail.com
}

\begin{abstract}
Objective of this experience report is to address specific issues regarding standard SAP ERP implementation in a medical institution. Target Company is a state owned health care institution from Bosnia and Herzegovina. Report will treat selected issues which could trouble standard SAP ERP implementation trough predefined ERP implementation methodology for SAP ERP. This report presents observations/remarks based on experience of authors in particular SAP ERP implementation project in health care institution. Author's goal is to provide useful insight into "real life" standard ERP implementation and problems that arise. Also, it should provide useful information for all stakeholders involved in the process of ERP implementation in public health care sector.
\end{abstract}

Keywords: SAP ERP All-in-One, Health care information systems, Inventory management, Health care billing system.

\section{Introduction}

This industry report provides insight into an implementation of SAP ERP solution in a major medical institution in Bosnia and Herzegovina with several thousands of employees. The project was initiated by management of the hospital with purpose to eliminate the ineffectiveness of the current information system. Analysis of the current financial system and the list of new system requirements have been prepared by an external consulting company. This was required for announcing a public tender for selection of the ERP software solution integrator. After several months of tender procedure and assessment of the best vendor (price was eliminatory criteria, in accordance with the law), software integrator was selected. At the end, SAP All-inOne solution [1] was the preferred one. Relevant project information is presented in next sections.

As recommended by external consultants the main goals of the project were:

- $\quad$ To centralize the information system (create/provide a centralized database);

- To increase data integrity and consistency;

- To focus on accounting and financial department processes; 
- To improve drug warehouse management and billing system;

- To provide comprehensive and accurate reports for top management;

The project incorporated five SAP modules: FI (Finance), CO (Controlling), MM (Material management), SD (Sales and Distribution) and HR (Human resources). SAP integrator offered a team of seven SAP solution consultants, including one SAP system administrator. In addition, two consultants (ABAP programmers) were teamed up for specific ABAP developments. During the implementation it became clear that the number of consultant was inadequate, given the project scope and specific demands in medical service industry.

\section{Situation before SAP Implementation}

The hospital is an institution that devotes considerable resources in upgrading its primary domain and activities by investing in specialized diagnostic and medical equipment. However, this is not the case with ICT. No substantial investments were made in information system improvements in the last twenty years. Moreover, parts of existing hospital IT infrastructure were damaged during the war (1992-1995).The example which reflects this initial situation is that network between Clinics was nonexistent of network infrastructure between Clinics which is the basic assumption for any SAP implementation

Due to the law regulations, the hospital was obliged to have certain software solutions, mainly disintegrated applications aimed at specific business domain. This software landscape contained dozen applications developed by one or few individuals. There was no recognized company behind them. All applications were technically isolated instances, mostly installed only on local machines with no server-client architecture. All master and transactional data were stored on a single machine. Only network connected application was accounting (General ledger) application (terminals), which was implemented in the eighties. The final (consolidated) reports, made by manual data collecting from various applications, were presented to the steering committee. The process of preparing reports took between five to ten days. Microsoft Excel sheets were being (physically on USB) passed from department to department. The most representative example of their legacy IT architecture was the billing system. Each PC machine had application installed for specific billing types (7 types/scenarios of billing); there were seven local machines each responsible for the specific billing scenario.

\section{Implementation and Its Specific Features}

The implemented system was standard SAP ERP All-in-One. Since it was not specialized health care solution, additional industry-specific functionalities needed to be developed to fulfill basic client's needs. They were mostly related to the processes of Materials Management and Sales and Distribution. 
In the following sections we will discuss main issues faced during SAP ERP implementation in two mentioned modules: MM (Materials Management) and SD (Sales and Distribution).

\subsection{Sales and Distribution Functionality and Obstacles}

Standard functionalities of SAP ERP SD include handling of sales and distribution. The main activities are the sales order handling, and the distribution of shipments to customers. In addition the billing process, customer invoice, delivery, and risk management are responsibility of the SD module. The Sales and Distribution (SAP $\mathrm{SD}$ ) consists of all master data, system configuration, and transactions to complete the Order. The billing process is important for every Health Institution, hence it was recognized that SD module is necessary to be installed in the hospital.

Particular attention was paid on the billing department which bills all medical and non-medical services provided by the institution. Billing department employs of 20 people, who create over 15000 invoice documents (billing documents) each month. This number of invoices does not pose a problem for SAP SD module. The real issue was the complexity of the invoicing process. As the previous billing software was outdated, complex billing affected process of collecting receivables negatively, as billing department was constantly in delay of two months when invoicing services provided to patient.

The process of invoicing can be divided into three stages, as shown in the Figure 1:

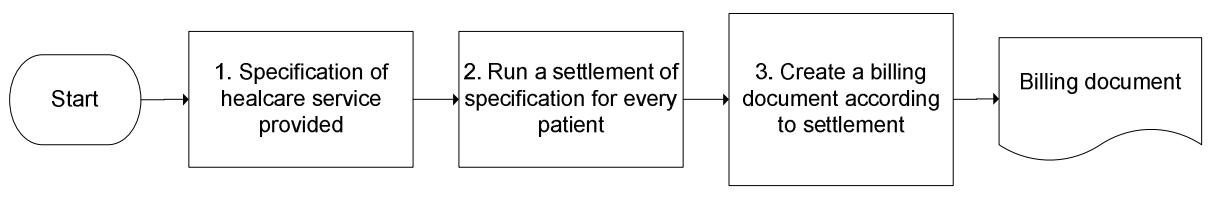

Fig. 1. Process of invoicing in the hospital

All processes shown in the diagram above were conducted in separate programs (software). This meant that for each operation, point cleric was required to re-enter data. The reliability of information over this life cycle was not guaranteed as the process was manual. In addition, billing of health services was carried out separately for different types / groups of patients; 7 types in total. All these different types have a special logic of calculation for health services, which was necessary to be realized and implemented through the implementation of the SAP SD module.

Issues and problems arose during SAP SD implementation:

a) The first detected problem was that the standard SAP system does not contain functionalities which would adequately handle the first process in billing system's life cycle - creation of specifications for health services provided to a patient. The suggestion given by SD consultants was not implementing this particular process with SAP system, and leaving it for the eventual second phase of the project, which was 
the implementation of the full SAP Healthcare solution. This suggestion was rejected by the steering committee. The decision was to develop this functionality using ABAP programming. . Add-on for standard SAP SD module had to be made, which would cause a number of changes in core SD processes (sales orders creation and billing documents creation).

At first, decision seemed reasonable, but later it proved to be one of the biggest mistakes made by SAP project manager. This generated other problems, such as dramatic increase in number of new users and SAP licenses needed for employees who would work on the data input for specification of health services and billing. It became apparent that the project cost was very close to violate the budget.

b) Another big issue raised was that the process of specification orders of healthcare services and pricing calculation was not standardized. The procedures and rules for price calculation differed between the clinics, so there were over 10 "QS procedures" which greatly hampered the first phase of SAP project - creation of Blueprints. Blueprint phase, which was planned to last for maximum of one month, was extended to a 80 days, due to unsynchronized billing processes and defined billing procedures in the hospital (once again - each clinic has its own procedure). Also, each clinic has its own working practice, which put additional strain on system integrator. Although the Blueprints phase represents the very first phase of the SAP project according to the ASAP methodology, it was clear that the period of 4 months for full implementation and go live were insufficient. All of this put enormous pressure on consultants.

c) The process of master data migration, and import preparation on the test system, discovered a lot of irregularities of the previous billing system procedure. It was possible for the same patient to have four different codes (system ID) depending on the type of service for which he was billed.

By the end of the project, along the standard ones, the following non-standard functionalities were implemented (developed as add-on):

1. Creation of specification of healthcare services (two type and transactions for ambulant and hospital use);

2. Creation of advance payment for hospital and ambulant use;

3. Automated process for creation of sales order referenced on healthcare specification;

4. Automation of price calculation depending on a patient's status;

5. Development of many $\mathrm{Z}$ reports (add on development on standard SAP).

\subsection{Material Management Functionality and Obstacles}

From the beginning of the project it was apparent that the drug warehouse of the hospital will be the most demanding part of the whole project. With annual turnover 
around 40 million EUR it was the major consumer of the hospital's budget. New, efficient inventory management system was essential to the "profitability" of the drug warehouse.

SAP ERP All-in-One was chosen to replace the old legacy system that was in use. This existing system was specifically designed and developed for the drug warehouse with features adjusted to their specific needs. The system was in use for over 12 years and was improved/upgraded during this time. However, the system operated only in this organizational unit. At the end of each month, accounting department was provided with the reports (goods receipt, goods issue, goods transfer, inventory status etc.) from the system, which contained no detailed analytics in itself.

The SAP module which covers processes of clinical drug warehouse is called SAP MM (Material management) module. All the issues that standard SAP MM module faces during implementation phase are stated in the rest of this section.

a) Adequate inventory is generally defined as basic stock plus safety stock. Inventory which is to big is a leading cause for unsatisfactory cash flow. On regular basis, hospital's drugstore had to put more of its cash into the purchase of medicaments in order to meet its obligations, especially in the case of the wholesaler who was located outside of Bosnia and Herzegovina. In theory, SAP ERP All-in-One solution provides the MRP function which covers the safety stock. The idea is to alert the responsible person when a group of materials or a material is close to the minimum of inventory stock, and suggest the number of units that should be purchased in order to meet the optimal stock. However, in practice, system provided alerts only when the responsible employee wanted to order particular material. Unfortunately, it was possible that the material is already under the safety stock red line. This was frustration for both, SAP consultants, and hospital warehouse management.

b) The complete database of pharmacy's inventory needed to be implemented according to the generic molecules, as it ensures standardization across health systems.

c) Even though it was not defined in the scope of the project, it was necessary to fulfill all pharmacy standards regarding pharmacy business proposed by law. All generics \& their particular brands, batches, used by the hospital had to be maintained in a database with the appropriate commercial terms (commercial name, pharmacy agency approval number etc). At first, the main challenge for the SAP integrator and consultants was implementation of ATC/DDD standardization for medicaments. The ATC/DDD system classifies all therapeutic drugs and provides a tool for drug utilization research in order to improve quality of drug use. In the ATC classification system, drugs are divided into different groups according to the organ or the system on which they act and their chemical, pharmacological and therapeutic properties. Drugs are classified into 14 main groups / classes [2], [3]. Each of the main classes contains hundreds of subgroups which include hundreds of groups itself. At the end, there was a need for thousands of predefined material groups in SAP MM. This 
approach was overly complicated so the integrator decided to implement the SAP Classification system for clinic drug warehouse.

The Classification system in SAP for clinical drug warehouse was, at the beginning, seen as blueprint of ATC/DDD classification. However, implementation/situation became more complex. The Classification system in SAP does not only depend on the definition of the ATC/DDD, but also on costing procedures and reporting (which was wanted to get as reports from classification). In addition, assuming that SAP classification needs to meet all, by law, proposed legislation, SAP integrator defined set of characteristics for each material. After few kick off meetings integrator came up with those classification characteristics; presentation/package (box, pills, mg etc.), aggregate state, product hierarchy grouping (ATC/DDD) and profit centers. All of these had to be set as characteristics of the material classification. After months of preparation of all materials (over 30000 records) for import into the system with all the necessary classification characteristics, it became apparent that such a big number of characteristics would be hard to maintain. Performance of drugstore as whole was seriously jeopardized by the time required to complete the entire process of maintaining material master data. At the end, this SAP classification system was refused by the hospital's drugstore.

d) The lack of batch management and shelf life - The main and very important point of any drug warehouse is the batch management. All pharmacy products are managed by the batch and the shelf life. Those are critical factors for managing the inventory efficiently. The standard SAP ERP provides fields (after activation) for those material attributes, but, once again, it did not work properly in practice. The standard SAP ERP does not provide ad-hoc integration of bar code reader technology, so the only option was to extend the project's scope and to develop the specific solution for bar code technology. Moreover, integrator was required to build up good receipt transaction in SAP (MIGO transaction) in order to support goods receipt process supported by code readers. This particular development increased the project's costs.

Another issue was the shelf life of materials. The proposition from hospital drug warehouse management was to create inventory management system where medicaments with closest expire date would be suggested for goods issue. However, this was not a feature of the standard SAP ERP All-in-One. It was only possible to create a new report where all medicaments would be listed by the expiry date criteria. However, this was not satisfactory for clinical drug management because their employees required too much time to operate with particular report.

e) Standardization hierarchy for equipment (medical as well as non- medical) The complete database of non-pharmacy inventory like equipment, linen, stationary, sundry items etc. was imported in SAP. Also, the database of the approved suppliers for each item was maintained with relevant commercial terms \& specifications. However, as with the ATC/DDD standardization, the proposed solution for standardization of the equipment (class/subclass/group) was impractical for daily usage. 
f) Goods issue of material was not linked to the specification of healthcare services for a patient (SAP SD module) - One of the major issues for the SAP integrator was implementation of fully automated goods issue process. At the beginning the idea was to blueprint the real life scenario. That is, medicament was supposed to be issued when given to patient. However, in reality, due to the lack of infrastructure (no available network) and because of the license issue (management of clinic found it too expensive SAP), process was delayed 7 days at average. So, medicament was issued to a patient from the department drug storage location, but transaction was written on a paper. After 7 days, when the list of medicaments issued is usually over 50 pages long, it is provided to employees in central hospital drugstore, and then imported into SAP. This was undesirable process for employees who import those lists weekly into SAP. In addition, dozens of mistake were made each week due to manual data import into SAP system.

\section{Conclusion}

Implementation of the standard SAP system in health care environment results in specific issues and problems during the implementation of SAP ERP SD and MM modules. The main problems encountered explained for SD and MM module are:

- Standard SAP SD module does not fit health care institution billing needs too much development is needed, which is not good for maintenance and support;

- $\quad$ SAP ERP SD is not intended to cope with patient master date (huge load of data);

- The Standard material group in MM is not sufficient to describe ATC/DDD standardization;

- SAP material classification is too complex when it respects all attributes needed for maintaining drug material master data.

This leads to the general conclusions regarding implementation of standard SAP system in health care institution:

- Standard SAP ERP system does not support specific Healthcare processes, so a lot of custom development (i.e. "Z" programs) must be made to fulfill customer's requirements. It is a risky decision, because it boosts system maintenance cost;

- SAP All-in-One is not capable to meet all the health care institution requirements in a satisfactory way without SAP Healthcare solution, i.e. SAP ERP is tailored to fit standard business processes present in majority of business domains. However, only SAP Healthcare industry specific solution addresses all specific requirements for healthcare institution. 


\section{References}

1. SAP Business All-in-One, http://www.sap.com/sme/solutions/businessmanagement/ businessallinone/index.epx

2. WHO: The Anatomical Therapeutic Chemical Classification System with Defined Daily Doses (ATC/DDD), http: / / www. who.int/classifications/atcddd/en/

3. Anatomical Therapeutic Chemical Classification System, http://en.wikipedia.org/wiki/Anatomical_Therapeutic_ Chemical_Classification_System 